PROCEEDINGS OF THE

AMERICAN MATHEMATICAL SOCIETY

Volume 131, Number 12, Pages 3893-3899

S 0002-9939(03)06957-0

Article electronically published on February 28, 2003

\title{
A GENERALIZATION OF FILLIMAN DUALITY
}

\author{
GREG KUPERBERG
}

(Communicated by John R. Stembridge)

\begin{abstract}
Filliman duality expresses (the characteristic measure of) a convex polytope $P$ containing the origin as an alternating sum of simplices that share supporting hyperplanes with $P$. The terms in the alternating sum are given by a triangulation of the polar body $P^{\circ}$. The duality can lead to useful formulas for the volume of $P$. A limiting case called Lawrence's algorithm can be used to compute the Fourier transform of $P$.

In this note we extend Filliman duality to an involution on the space of polytopal measures on a finite-dimensional vector space, excluding polytopes that have a supporting hyperplane coplanar with the origin. As a special case, if $P$ is a convex polytope containing the origin, any realization of $P^{\circ}$ as a linear combination of simplices leads to a dual realization of $P$.
\end{abstract}

\section{INTRODUCTION}

If $P \subset \mathbb{R}^{d}$ is a polytopal region, let $[P]$ denote the restriction of Lebesgue measure to $P$. The measure $[P]$ can also be called the characteristic measure, by analogy with the characteristic function. We consider measures rather than functions so that if $P$ and $Q$ are two regions with disjoint interiors, then

$$
[P \cup Q]=[P]+[Q]
$$

even if $P$ and $Q$ are not disjoint at the boundary. If $P$ is convex and the origin lies in its interior, then $P$ admits a polar body $P^{\circ}$. Say that a polytope $P$, convex or not, is codegenerate if one of its facets is coplanar with the origin. Every noncodegenerate simplex $\Delta$ admits a polar simplex $\Delta^{\circ}$. Let $\mathcal{T}$ be a triangulation of $P^{\circ}$ by noncodegenerate simplices. In this circumstance Filliman [2] showed that

$$
[P]=\sum_{\Delta \in \mathcal{T}}(-1)^{\sigma(\Delta)}\left[\Delta^{\circ}\right],
$$

where $\sigma(\Delta)$ is a certain sign function. This formula is called Filliman duality. Figure 1 shows an example: a triangulation of a pentagon $P$ and the dual realization of $P^{\circ}$ as a triangle with two smaller triangles subtracted.

Two special cases of Filliman duality are notable. First, if each simplex $\Delta \in \mathcal{T}$ shares vertices with $P^{\circ}$, then $\Delta^{\circ}$ shares supporting hyperplanes with $P$. If $P$ has few vertices and many sides, then it is reasonable to compute its volume as the sum of the volumes of the simplices in a triangulation. But if $P$ has many vertices and few sides, it is more efficient to use a triangulation of $P^{\circ}$ via Filliman duality. Second,

Received by the editors December 23, 2001 and, in revised form, June 24, 2002 and July 10, 2002.

2000 Mathematics Subject Classification. Primary 52B45.

The author was supported by NSF grant DMS \#0072342. 

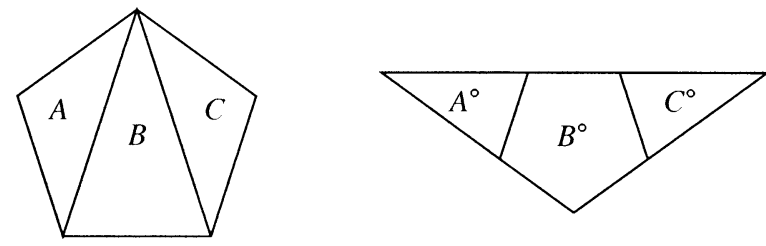

$$
[P]=[A]+[B]+[C] \quad\left[P^{\circ}\right]=\left[B^{\circ}\right]-\left[A^{\circ}\right]-\left[C^{\circ}\right]
$$

FiguRE 1. A triangulation of a pentagon and its Filliman dual.

if $\mathcal{T}$ is the cone of a triangulation of $\partial P^{\circ}$, then as the apex of the cone converges to the origin, each dual simplex $\Delta^{\circ}$ with $\Delta \in \mathcal{T}$ converges to an affine orthant emanating from a vertex of $P$. Thus we an express $[P]$ as an alternating sum of such orthants. This limiting case of Filliman duality is called Lawrence's algorithm [4]. It is useful not only for finding the volume of $P$, but also for computing the Fourier transform of $[P]$.

In this note we extend Filliman duality to an involution on noncodegenerate, integral, polytope measures on $\mathbb{R}^{d}$. More precisely, let $\mathcal{A}$ be the abelian group of signed measures $\mu$ on $\mathbb{R}^{d}$ of the form

$$
\mu=\sum_{i=1}^{n} \alpha_{i}\left[P_{i}\right],
$$

where each $P_{i}$ is a noncodegenerate polytope and $\alpha_{i} \in \mathbb{Z}$. If $\Delta$ is a noncodegenerate simplex, let $\sigma(\Delta)$ be the number of supporting hyperplanes of $\Delta$ that separate it from the origin. Let $\mathcal{D} \subset \mathcal{A}$ consist of measures of the form $[\Delta]$.

Theorem 1. The involution $\Phi: \mathcal{D} \rightarrow \mathcal{D}$ defined by

$$
\Phi([\Delta])=(-1)^{\sigma(\Delta)}\left[\Delta^{\circ}\right]
$$

extends uniquely to an automorphism $\Phi: \mathcal{A} \rightarrow \mathcal{A}$.

The following corollary captures the original Filliman duality as part of the involution $\Phi$ :

Corollary 2. If $P$ is a convex polytope containing the origin in its interior and

$$
[P]=\sum_{i=1}^{n} \alpha_{i}\left[\Delta_{i}\right]
$$

for simplices $\Delta_{1}, \ldots, \Delta_{n}$, then

$$
\left[P^{\circ}\right]=\sum_{i=1}^{n}(-1)^{\sigma(\Delta)} \alpha_{i}\left[\Delta_{i}^{\circ}\right] .
$$

Note that our involution $\Phi$ is not the same as the Euler involution on the polytope algebra defined by McMullen [6]. Nonetheless, the group $\mathcal{A}$ and the map $\Phi$ on it are ultimately a disguised specialization of a known involution in valuation theory, the polarity map on cones (see Section 4). Thus the real significance of Theorem 1 and its proof is not that it describes an essentially new involution, as the author once thought, but rather that it relates three distinct constructions in combinatorial geometry: Filliman duality, valuation theory, and the stellar subdivision theorem. 


\section{Proof of Theorem 1}

In this section we assume that all simplices and other polytopes are noncodegenerate except where noted.

We will consider signed polytopes in order to absorb the sign that appears in duality for simplices. A signed polytope $P$ is a polytope together with a formal sign, either + or - . Characteristic measures on signed polytopes are defined by the rule $[-P]=-[P]$. If $\Delta \subset \mathbb{R}^{d}$ is a simplex with positive sign, we define

$$
\Delta^{*}=(-1)^{\sigma(\Delta)} \Delta^{\circ}, \quad(-\Delta)^{*}=-\left(\Delta^{*}\right) .
$$

Also we recall the definition of $\Delta^{\circ}$. If $\Delta$ has vertices $v_{0}, \ldots, v_{d}$, then $\Delta^{\circ}$ is bounded by the hyperplanes $H_{v_{0}}, \ldots, H_{v_{d}}$, where for any vector $v, H_{v}$ is defined as

$$
H_{v}=\{w \mid\langle w, v\rangle=1\}
$$

Let $\mathcal{B}$ be the abelian group freely generated by simplex measures $[\Delta]$. By summing the terms of each element in $\mathcal{B}$, we obtain a homomorphism $\pi: \mathcal{B} \rightarrow \mathcal{A}$. It is surjective because every polytopal region can be tiled by simplices. The involution $\Phi$ extends tautologically to $\mathcal{B}$. Theorem 1 then asserts that $\Phi$ preserves $\operatorname{ker} \pi$. In order to prove this we first give a characterization of the kernel. The characterization depends on a refinement of the stellar subdivision due to M. H. A. Newman [5], 7].

Theorem 3 (Newman). Any two triangulations of a polytope $P \subset \mathbb{R}^{d}$ are equivalent under stellar moves applied on edges.

Corollary 4. The kernel ker $\pi$ is generated by the relators

$$
[\Delta]-\left[\Delta_{1}\right]-\left[\Delta_{2}\right]
$$

where $\Delta$ is a simplex tiled by two simplices $\Delta_{1}$ and $\Delta_{2}$.

Proof. Let $\mathcal{I}$ be the subgroup of $\mathcal{B}$ generated by the relators. Clearly $\mathcal{I} \subseteq$ ker $\pi$; we wish to show that $\operatorname{ker} \pi \subseteq \mathcal{I}$. Assume a general linear dependence of simplices

$$
\sum_{i=1}^{n} \alpha_{i}\left[\Delta_{i}\right]=0
$$

in $\mathcal{A}$. Equivalent, in $\mathcal{B}$

$$
\sum_{i=1}^{n} \alpha_{i}\left[\Delta_{i}\right] \in \operatorname{ker} \pi
$$

The union of the simplices,

$$
P=\bigcup_{i=1}^{n} \Delta_{i}
$$

is a compact polytopal region in $\mathbb{R}^{d}$. It admits a triangulation $\mathcal{T}$ that refines each simplex $\Delta_{i}$. Let $\mathcal{T}_{i}$ be the restriction of $\mathcal{T}$ to the simplex $\Delta_{i}$. By Theorem 3 , the triangulation $\mathcal{T}_{i}$ can be obtained from the tautological triangulation of $\Delta_{i}$ by itself by stellar moves applied to edges. A stellar move on some edges $e$ can be effected by dividing each simplex containing $e$ into two simplices, the geometric move captured by the relator. Therefore

$$
\left[\Delta_{i}\right]-\sum_{\Delta \in \mathcal{T}_{i}}[\Delta] \in \mathcal{I}
$$


in $\mathcal{B}$. At the same time, equation (1D) implies that

$$
\sum_{i=1}^{n} \alpha_{i} \sum_{\Delta \in \mathcal{T}_{i}}[\Delta]=0
$$

in $\mathcal{B}$, since each simplex in $\mathcal{T}$ must be covered a total of 0 times. Therefore ker $\pi \subseteq \mathcal{I}$, as desired.

Remark. Call the move of dividing a simplex into two an elementary dissection. An interesting fact closely related to Corollary 4 is that any two simplicial dissections of a polytopal region are connected by elementary dissections.

Proof of Theorem 1. In light of Corollary 4, we only need to check that $\Phi$ preserves an elementary dissection of a simplex. If $v_{0}, v_{1}, \ldots, v_{d}$ are the vertices of a simplex $\Delta$, we let the word $v_{0} v_{1} \cdots v_{d}$ denote $\Delta$ if

$$
v_{1}-v_{0}, v_{2}-v_{0}, \ldots, v_{d}-v_{0}
$$

is a positive basis of $\mathbb{R}^{d}$, and otherwise we let it denote $-\Delta$. If $w_{i}$ is the vertex of $\Delta^{*}$ opposite to the hyperplane $H_{v_{i}}$, it follows that

$$
\Delta^{*}=(-1)^{d} w_{0} w_{1} \cdots w_{d} .
$$

Suppose that $x_{1}, x_{2}$, and $x_{3}$ are three collinear points in $V$, and suppose that $v_{0}, \ldots, v_{d-2}$ are $d-1$ other points affinely independent from any two of $x_{0}, x_{1}$ and $x_{2}$. Then

$$
\left[v_{0} \cdots v_{d-2} x_{1} x_{2}\right]+\left[v_{0} \cdots v_{d-2} x_{2} x_{3}\right]+\left[v_{0} \cdots v_{d-2} x_{3} x_{1}\right]=0
$$

expresses an elementary dissection. Applying $(-1)^{d} \Phi$ to both sides produces

$$
\left[w_{0} w_{1} \cdots w_{d-2} y_{2} y_{1}\right]+\left[w_{0} w_{1} \cdots w_{d-2} y_{3} y_{2}\right]+\left[w_{0} w_{1} \cdots w_{d-2} y_{1} y_{3}\right]=0
$$

Here each point $w_{i}$ lies in the hyperplanes $H_{v_{j}}$ for $i \neq j$ and in the hyperplanes $H_{x_{j}}$ for all $j$. Each point $y_{i}$ lies in the hyperplane $H_{v_{j}}$ for all $j$ and in the hyperplane $H_{x_{i}}$. Evidently equation (3) is the same equation up to sign as equation (21). Figure 2 shows an example.
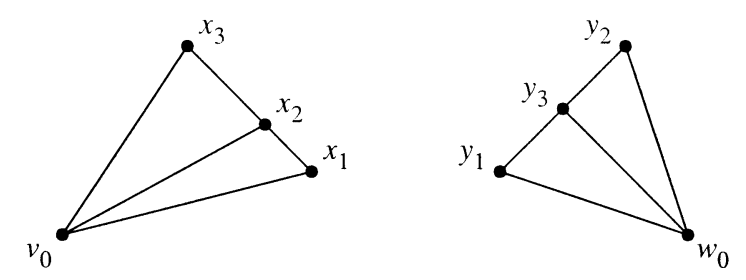

Figure 2. An elementary dissection and its dual. 


\section{Proof of Corollary 2 and examples}

Corollary 2 follows immediately from Theorem 1 and the following proposition.

Proposition 5. If $P \subset \mathbb{R}^{d}$ is a convex polytope that strictly contains the origin, then $\Phi([P])=\left[P^{*}\right]$, the polar body of $P$.

Proof. We first assume that any $d$ vertices of $P$ are linearly independent, or equivalently affinely independent from the origin. Let $\mathcal{T}$ be a triangulation of $P$ with no vertices in the interior of $P$. We claim, first, that any point $w$ in the interior of $P^{*}$ is covered by the dual of exactly one simplex. A unique simplex $\Delta_{0} \in \mathcal{T}$ contains the origin. The inclusions $0 \in \Delta_{0} \subset P$ imply that $\Delta_{0}^{*} \supset P^{*}$, so $\Delta^{*}$ covers $w$. If $\Delta \in \mathcal{T}$ is another simplex, then there exists a vertex $v$ of $\Delta$ which is separated from the origin by the opposite face of $\Delta$. It follows that $\Delta^{*}$ is separated from the origin by the hyperplane $H_{v}$. Since $v$ is also a vertex of $P, H_{v}$ is a supporting hyperplane of $P^{*}$. Therefore $H_{v}$ separates $\Delta^{*}$ from $P^{*}$ and $\Delta^{*}$ does not contain $w$. This establishes the first claim.

We claim, second, that if $w$ is the exterior of $P^{*}$, there exists a triangulation $T$ of $P$ such that $w$ is not covered by $\Delta^{*}$ for any $\Delta \in T$. There exists a vertex $v$ of $P$ such that the hyperplane $H_{v}$ separates $w$ from $P^{*}$. Let $\mathcal{T}$ be a fan triangulation all of whose simplices contain $v$. If $\Delta \in \mathcal{T}$, then $H_{v}$ separates $\Delta$ from $w$, as desired. The two claims together with Theorem 1 establish the proposition under the independence assumption on $P$.

Finally we assume that $P$ is arbitrary. The argument so far establishes the proposition for the polytope $P-v$ for a dense set of vectors $v$ in the interior of $P$. Namely $v$ can be any point that does not lie on a hyperplane affinely spanned by vertices of $P$. But if $v$ is in the interior of $P$, then $P-v$ has a noncodegenerate triangulation whether or not $v$ lies on such a hyperplane. It follows that $\Phi([P-v])$ varies continuously in a neighborhood of $v$. Thus the truth of the proposition for a dense set of $v$ implies its truth for all $v$ in the interior of $P$. In particular, the proposition holds for $v=0$.

We conclude with two examples of polygonal regions in the plane and their images under $\Phi$. Figure 3 shows the dual of a square with corners $(1,-1),(3,-1),(1,1)$, and $(3,1)$. The dual is a negative measure in the interior of a kite shape. Finally Figure 4 shows the dual of a square with corners $(1,1),(1,2),(2,1)$, and $(2,2)$. The dual is the difference (in the sense of signed measures) between two triangles with disjoint interiors.
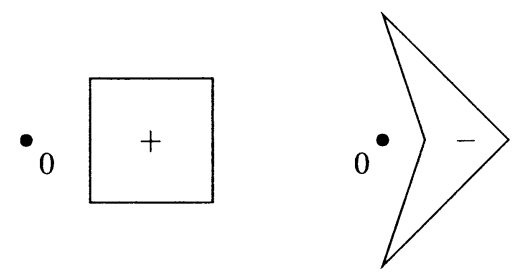

FiguRE 3. A square offset to the right and its dual. 

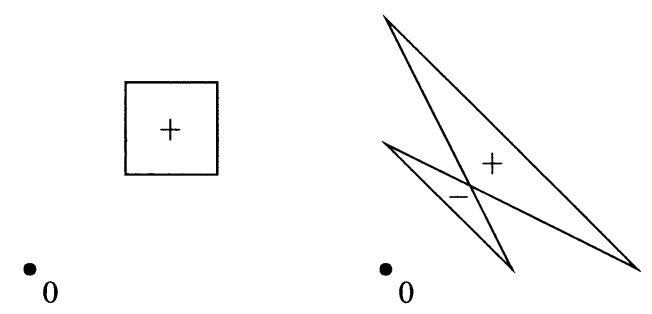

Figure 4. A square offset diagonally and its dual.

\section{INVOLUTIONS ON CONES}

In response to the first version of this article, Alexander Barvinok suggested that Theorem 1 is related to the fact that the polarity involution $\Pi$ on spherical convex polytopes extends to a valuation. In this section we show, in outline, that $\Phi$ becomes a restriction of $\Pi$ after suitably mapping measures on $\mathbb{R}^{d}$ to functions on the sphere $S^{d}$.

Let $\mathcal{C}$ be the abelian group of integer-valued functions on $S^{d}$ spanned by the characteristic functions of closed (or equivalently open) spherical polytopes of any dimension $\leq d$. If $P \subset S^{d}$ is closed and convex, then it admits a polar dual

$$
P^{\circ}=\{x \mid \forall y \in P,\langle x, y\rangle \geq 0\},
$$

where the inner product uses the defining embedding $S^{d} \subset \mathbb{R}^{d+1}$. Also, if $P \subset S^{d}$, let $\chi_{P}$ be the characteristic function of $P$. The following result in combinatorial geometry is known but unattributed (see Barvinok [1] and Lawrence [3]):

Theorem 6. The polarity map

$$
X_{P} \mapsto \chi_{P^{\circ}}
$$

extends to an involution $\Pi: \mathcal{C} \rightarrow \mathcal{C}$.

Now identify $\mathbb{R}^{d}$ with the open upper hemisphere in $S^{d}$ by stereographic projection. Let $\mathcal{D} \subset \mathcal{C}$ be the set of those functions $f$ such that:

(1) $f$ is supported in $\mathbb{R}^{d}$,

(2) $f \circ \sigma$ is defined using noncodegenerate polytopes, and

(3) $f \circ \sigma$ is radially left-continuous as a function on $\mathbb{R}^{d}$, meaning that for all $v \in \mathbb{R}^{d}$

$$
\lim _{t \rightarrow 1^{-}}(f \circ \sigma)(t v)=(f \circ \sigma)(v) .
$$

It is not hard to show that the class $\mathcal{D}$ is spanned by (the characteristic functions of) closed convex polytopes with the origin in their interiors. Thus $\mathcal{D}$ is invariant under the polarity involution $\Pi$.

It is also not hard to show that every measure $\mu \in \mathcal{A}$, if interpreted as an element of $L^{1}\left(\mathbb{R}^{d}\right)$, is represented by a unique radially left-continuous function $f \in \mathcal{D}$. This identifies $\mathcal{A}$ with $\mathcal{D}$. Again, because $\mathcal{D}$ is spanned by closed convex polytopes with the origin in their interiors. Because both maps $\Phi$ and $\Pi$ are the polarity transformation on this class, the two maps are identified as well.

Note that the class $\mathcal{D}$ extends to a slightly larger class $\overline{\mathcal{D}}$ spanned by all convex polytopes $P$ in the closed upper hemisphere in $S^{d}$ which contain the origin (not necessarily in the interior). The class $\overline{\mathcal{D}}$ is also invariant under the polarity involution 
$\Pi$. Indeed $\overline{\mathcal{D}}$ is the closure of $\mathcal{D}$ with respect to a natural topology on $\mathcal{C}$, namely the one induced by the Hausdorff topology on closed subsets of $S^{d}$. Moreover $\Pi$ is continuous with respect to this topology. Thus the restriction of $\Pi$ to $\overline{\mathcal{D}}$ expresses all codegenerate limiting cases of Filliman duality, such as Lawrence's algorithm.

\section{ACKNOWLEDGMENTS}

We would like to thank Alexander Barvinok, Jesus De Loera, Yael Karshon, Colin Rourke, and Günter Ziegler for useful discussions.

\section{REFERENCES}

[1] Alexander Barvinok, Convexity, to appear in Graduate Studies in Mathematics, 2002.

[2] Paul Filliman, The volume of duals and sections of polytopes, Mathematika 39 (1992), no. 1, 67-80. MR 93g:52005

[3] Jim Lawrence, Valuations and polarity, Discrete Comput. Geom. 3 (1988), no. 4, 307-324. MR 90b:52001

[4] , Polytope volume computation, Math.Comp. 57 (1991), no. 195, 259-271. MR 91j:52019

[5] W. B. R. Lickorish, Simplicial moves on complexes and manifolds, Geom. Topol. Monogr. 2 (1999), 299-320, arXiv:math.GT/9911256. MR 2000j:57057

[6] Peter McMullen, The polytope algebra, Adv. Math. 78 (1989), no. 1, 76-130. MR 91a:52017

[7] M. H. A. Newman, A theorem in combinatorial topology, J. London Math. Soc. 6 (1931), $186-192$.

Department of Mathematics, University of California-Davis, Davis, California 95616

E-mail address: greg@math.ucdavis.edu 